\title{
THE BOUSSINESQ INTEGRABLE SYSTEM: COMPATIBLE LATTICE AND CONTINUUM STRUCTURES
}

\author{
ANASTASIOS TONGAS and FRANK NIJHOFF \\ Department of Applied Mathematics, University of Leeds, Leeds LS2 9JT, U.K.
}

(Received 23 February, 2004; accepted 20 July, 2004)

\begin{abstract}
We consider the discrete Boussinesq integrable system and the compatible set of differential difference, and partial differential equations. The latter not only encode the complete hierarchy of the Boussinesq equation, but also incorporate the hyperbolic Ernst equations for an Einstein-Maxwell-Weyl field in general relativity. We demonstrate a specific symmetry reduction of the partial differential equations, to a six-parameter, second order coupled system of ordinary differential equations, which is conjectured to be of Garnier type.
\end{abstract}

2000 Mathematics Subject Classification. 35Q58.

1. Introduction. One of the outstanding universal features of integrable nonlinear evolution equations is their appearance together with a structured infinite hierarchy of commuting flows, i.e. an infinite set of mutually compatible partial differential equations (PDE). This feature reflects the extremely high degree of symmetry hidden in the system, and it has been exploited in many ways leading to the intimate connection between the associated hierarchies on the one hand, and the representation theory of affine Lie algebras and loop groups, on the other hand, c.f. [1], [2] and references therein. However, the point of view that one single equation in the associated hierarchy possesses a dominant role is misguided and, in fact, one should consider the infinite set of PDEs altogether, which constitutes the hierarchy, as being the integrable system.

It is remarkable that there are situations in which the complete infinite hierarchy of flows of an integrable evolution equation, can be encoded in a single PDE. A prime example of such an equation was given recently in [3], and it is represented by a fourth order scalar PDE. The complete hierarchy of the Korteweg-de Vries (KdV) equation follows by systematic expansions from this PDE. In the present work we consider another hierarchy generating PDE, namely the one that encodes the complete hierarchy of the Boussinesq (BSQ) equation. This will be achieved by deriving, first, the compatible discrete integrable system.

The construction of the latter is based on the infinite-matrix structures, introduced in [4]. In particular we consider those structures which serve as a representation of the loop algebra built from the hierarchy of flows, of the second member in the Gel'fandDikii hierarchy, namely the Boussinesq equation [5]. The basic ingredient consists of a linear integral equation for (infinite) basic functions depending on a complex parameter and on the coordinates of the system. The coordinates can be chosen to be discrete as well as continuous, and exactly this freedom in the coordinate choice, is responsible for making the integral equations such a convenient tool to derive discrete integrable 
systems. This is the subject matter of Section 2, which ends up with a parameter family of discrete equations, namely the lattice BSQ equation.

In Section 3, the multi-dimensional consistency of the lattice BSQ equation is tested, by embedding the two dimensional lattice into a higher dimensional lattice. This aspect of the discussion forms a completion to the study of the lattice BSQ equation as performed in [6]. The fact that the evolution in the extended three dimensional lattice is well posed reveals, in an essentially algorithmic fashion, the Lax pair of the lattice BSQ equation. Section 4, proceeds with the derivation of the compatible set of differential-difference equations of the lattice BSQ equation. This will be achieved by imposing on the dependent variables an additional dependence on the continuous lattice parameters, in such a way that certain compatibility conditions are satisfied.

Sections 5 and 6, deal with the continuous compatible PDEs and ODEs, respectively. First, we present the generating PDE of the Boussinesq hierarchy, consisting of a two-parameter family of coupled fourth order system of PDEs, in two dependent and two independent variables. Next, we illustrate the connection of the latter PDEs with the Boussinesq hierarchy, using the Lax pair of the relevant PDEs, which was derived in the preceding sections. A prominent position, among the subsystems which are included into the solution space of the fourth order parameter family of PDEs, is possessed by a second order system. It is the heart of the Einstein's field equations for plane symmetric spacetimes, in the presence of certain massless fields. In the context of general relativity, the latter equations are known as the Ernst equations for an Einstein-Maxwell-Weyl field. Finally, we consider a particular type of similarity solutions of the system of PDEs under consideration, which are built from the solutions of a second order coupled system of ODEs, involving six free parameters. The latter may be considered to be associated with the hierarchies of the Painlevé VI equation.

The paper concludes with perspectives, where various avenues for future research are discussed.

2. The discrete Boussinesq system. In order to derive the lattice Boussinesq (BSQ) system, we shall use a linear integral equation of the form

$$
\boldsymbol{u}(k)+\int_{C} \mathrm{~d} \lambda(\ell) \frac{\boldsymbol{u}(k)}{k-\omega \ell}=\rho(k) \boldsymbol{r}(k)
$$

c.f. [5]. The vector $\boldsymbol{u}(k)$, has $i \in \mathbb{Z}$ entries, each of them taking values in the complex numbers, and $\boldsymbol{r}$ is a vector with entries given by $r_{i}=k^{i},(i \in \mathbb{Z})$. The variables $\ell$ and $k$ are complex, and $\omega$ is a primitive $N$-th root of unity, i.e. $\omega=\exp (2 \mathrm{i} \pi / N)$. The integrations in (1) are performed over an arbitrary contour $C$ in the complex $k$-plane, and over an arbitrary measure $\mathrm{d} \lambda(\ell)$. In principle, the only restrictions on the measure and the contour consist of the requirement of the existence and uniqueness of the solution of the integral equation (1), once the inhomogeneous term is specified. Along with the infinite vector $\boldsymbol{u}$, which solves the integral equation (1), we can introduce an infinite-component matrix potential $\boldsymbol{U}$, given by

$$
\boldsymbol{U}=\int_{C} \mathrm{~d} \lambda(\ell) \boldsymbol{u}(\ell)^{t} \boldsymbol{r}(\ell),
$$


where ${ }^{t} \boldsymbol{r}$ denotes the transposed vector of $\boldsymbol{r}$. The aim is to derive for the matrix potential $\boldsymbol{U}$, as well as for the vector potential $\boldsymbol{u}$, a set of (infinite-) matrix equations, by imposing on these objects a dependence on additional variables and certain transformation rules. The variables can be continuous as well as discrete. Adopting the latter case, we consider two operations, namely the maps

$\boldsymbol{u}(n, m, k) \mapsto \tilde{\boldsymbol{u}}(n, m, k)=\boldsymbol{u}(n+1, m, k), \quad \boldsymbol{u}(n, m, k) \mapsto \hat{\boldsymbol{u}}(n, m, k)=\boldsymbol{u}(n, m+1, k)$.

These maps have the interpretation of shifts in a two-dimensional lattice, but equally well of Bäcklund transformations generated by a transformation of the complex function $\rho(k)$, which is not specified yet. The transformation rules which are imposed on $\rho(k)$ are

$$
\begin{aligned}
& \rho(k ; p, q) \mapsto \tilde{\rho}(k ; p, q)=\frac{p+k}{p+\omega k} \rho(k ; p, q), \\
& \rho(k ; p, q) \mapsto \hat{\rho}(k ; p, q)=\frac{q+k}{q+\omega k} \rho(k ; p, q),
\end{aligned}
$$

where $p$ and $q$ are the complex lattice parameters, associated with the lattice variables $n$ and $m$, respectively. Using the linear integral equation (1), we may determine how $\boldsymbol{u}$ changes, under the action of the transformations (4). Restricting to the special case $N=3$, i.e. $\omega=\exp (2 \mathrm{i} \pi / 3)$, the equations which determine the change of $\boldsymbol{u}$, are given by

$$
\begin{aligned}
(p+\omega k) \tilde{\boldsymbol{u}}= & (p+\boldsymbol{\Lambda}-\tilde{\boldsymbol{U}} \boldsymbol{O}) \boldsymbol{u}, \\
(p+k)\left(p+\omega^{2} k\right) \boldsymbol{u}= & (p+\omega \boldsymbol{\Lambda})\left(p+\omega^{2} \boldsymbol{\Lambda}\right) \tilde{\boldsymbol{u}} \\
& -\boldsymbol{U}\left(\omega \boldsymbol{O}\left(p+\omega^{2} \boldsymbol{\Lambda}\right)+\omega^{2}\left(p+\omega^{2 t} \boldsymbol{\Lambda}\right) \boldsymbol{O}\right) \tilde{\boldsymbol{u}},
\end{aligned}
$$

together with the same equations by replacing $\tilde{\cdot}$ and $p$, with $\hat{\bullet}$ and $q$, respectively. The matrices $\boldsymbol{\Lambda}$ and ${ }^{t} \boldsymbol{\Lambda}$, define the operations of index-raising when multiplied from the left and from the right, respectively, i.e. $(\boldsymbol{\Lambda} \boldsymbol{u})_{i}=u_{i+1}$. The matrix $\boldsymbol{O}$ is a projection matrix which singles out the central element of the object which upon acts, i.e. $(\boldsymbol{O} \boldsymbol{u})_{i}=u_{0} \delta_{i 0}$, where $\delta_{i j}$ is the Kronecker delta symbol. Integrating (5) over the contour $C$ and the same measure $\mathrm{d} \lambda(\ell)$, we obtain the following nonlinear system of equations for the matrix potential $\boldsymbol{U}$

$$
\begin{aligned}
\tilde{\boldsymbol{U}}\left(p+\omega^{t} \boldsymbol{\Lambda}\right)= & (p+\boldsymbol{\Lambda}-\tilde{\boldsymbol{U}} \boldsymbol{O}) \boldsymbol{U} \\
\boldsymbol{U}\left(p+{ }^{t} \boldsymbol{\Lambda}\right)\left(p+\omega^{2 t} \boldsymbol{\Lambda}\right)= & (p+\omega \boldsymbol{\Lambda})\left(p+\omega^{2} \boldsymbol{\Lambda}\right) \tilde{\boldsymbol{U}} \\
& -\boldsymbol{U}\left(\omega \boldsymbol{O}\left(p+\omega^{2} \boldsymbol{\Lambda}\right)+\omega^{2}\left(p+\omega^{2 t} \boldsymbol{\Lambda}\right) \boldsymbol{O}\right) \tilde{\boldsymbol{U}} .
\end{aligned}
$$

Using the identity $\omega^{2}+\omega+1=0$, the above equations may be rewritten as follows

$$
\begin{aligned}
\tilde{\boldsymbol{U}}\left(p+\omega^{t} \boldsymbol{\Lambda}\right) & =(p+\boldsymbol{\Lambda}-\tilde{\boldsymbol{U}} \boldsymbol{O}) \boldsymbol{U} \\
\boldsymbol{U}\left(p^{2}-\omega p^{t} \boldsymbol{\Lambda}+\omega^{2}\left({ }^{t} \boldsymbol{\Lambda}\right)^{2}\right) & =\left(p^{2}-p \boldsymbol{\Lambda}+\boldsymbol{\Lambda}^{2}\right) \tilde{\boldsymbol{U}}+\boldsymbol{U}\left(p \boldsymbol{O}-\boldsymbol{O} \boldsymbol{\Lambda}-\omega^{t} \boldsymbol{\Lambda} \boldsymbol{O}\right) \tilde{\boldsymbol{U}} .
\end{aligned}
$$

From the above infinite set of relations for the components of the potential $\boldsymbol{U}$, we restrict ourselves to the equations evolving its $(0,0),(1,0)$ components from equation $(7 a)$ and the $(0,0)$ component from equation $(7 b)$, together with the same equations 


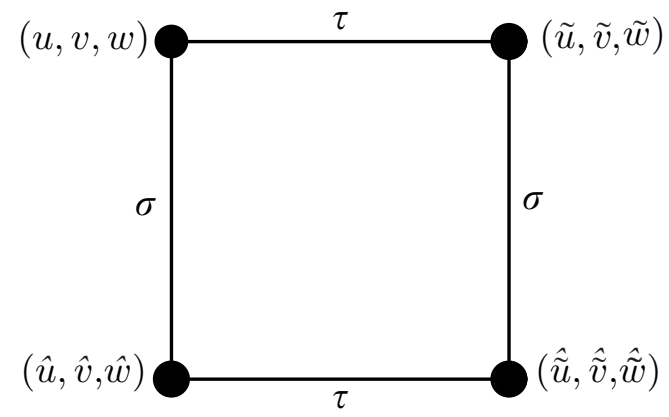

Figure 1. An elementary quadrilateral for the lattice Boussinesq system

by interchanging $\tilde{r}$ and $p$, with $\hat{\bullet}$ and $q$, respectively. A simple elimination process, using the discrete operations, leads to a closed system of equations for the variables $\left(U_{00}, U_{10}, U_{01}\right)$. The omitted latter set of discrete equations, obtains a simple form by introducing new dependent variables $(u, v, w)$, where

$$
\begin{aligned}
u & =U_{00}-(n p+m q), \\
v & =U_{10}-(n p+m q) U_{00}+\frac{1}{2} n(n+1) p^{2}+n m p q+\frac{1}{2} m(m+1) q^{2}, \\
w & =-\omega U_{01}-(n p+m q) U_{00}+\frac{1}{2} n(n-1) p^{2}+n m p q+\frac{1}{2} m(m-1) q^{2} .
\end{aligned}
$$

In terms of these new variables, the aforementioned set of partial difference equations $(\mathrm{P} \Delta \mathrm{E})$, reads

$$
\begin{aligned}
& \tilde{w}=u \tilde{u}-v, \\
& \hat{w}=u \hat{u}-v, \\
& w=u \hat{\tilde{u}}-\hat{\tilde{v}}+\frac{\tau-\sigma}{\hat{u}-\tilde{u}},
\end{aligned}
$$

where $\tau=p^{3}, \sigma=q^{3}$. Equations (9) will be referred in the following as the lattice BSQ system. They represent a multi-field system of equations living on an elementary quadrilateral, where the fields are assigned to the four vertices of the quadrilateral, and the associated parameters $\tau, \sigma$ are assigned to the edges, as shown in Figure 1. It should be noticed that for the Cauchy problem on a staircase, we should impose on an elementary plaquette the initial values on $(u, v, w),(\tilde{u}, \tilde{v}),(\hat{u}, \hat{v})$ only, since from these data and equations (9) we determine uniquely the values $\tilde{w}, \hat{w}$ and $(\hat{\tilde{u}}, \hat{\tilde{v}}, \hat{\tilde{w}})$.

3. Three dimensional consistency of the lattice Boussinesq. The lattice BSQ system (9), represents a compatible parameter family of $\mathrm{P} \Delta \mathrm{Es}$, in the sense that it can be embedded consistently in a multidimensional lattice, on which the evolution is well posed [7]. Recently, this property has been successfully exploited for quadrilateral scalar lattice equations to obtain a classification of the latter [8].

In order to illustrate the above statement, we first extend the lattice into a third dimension by introducing a new lattice variable $l$ associated with a new shift ${ }^{-}$, and a new complex parameter $\zeta$. Next we impose the system of equations (9) on all six faces of any elementary cube, as shown in Figure 2. Specifically, on the three faces adjacent 




Figure 2. Three dimensional consistency of the lattice Boussinesq system

to the vertex where the value $(u, v, w)$ is assigned, we have

$$
\begin{array}{ll}
\tilde{w}=u \tilde{u}-v, & w=u \hat{\tilde{u}}-\hat{\tilde{v}}+\frac{\tau-\sigma}{\hat{u}-\tilde{u}}, \\
\hat{w}=u \hat{u}-v, & w=u \overline{\tilde{u}}-\overline{\tilde{v}}+\frac{\tau-\zeta}{\bar{u}-\tilde{u}}, \\
\bar{w}=u \bar{u}-v, & w=u \overline{\hat{u}}-\overline{\hat{v}}+\frac{\sigma-\zeta}{\bar{u}-\hat{u}},
\end{array}
$$

and a similar set of equations on the remaining three faces. It is supposed that opposite edges on any face, carry the same parameter $\tau, \sigma$ or $\zeta$.

Let us now impose the following initial values

$$
(u, v, w), \quad(\tilde{u}, \tilde{v}), \quad(\hat{u}, \hat{v}), \quad(\bar{u}, \bar{v})
$$

to the vertices depicted with • in Figure 2. Using from (10) those equations which involve the shift of the variable $w$ in one direction, and taking into account the relevant compatibility conditions, we can determine how $u$ and $w$, are shifted in two directions. The corresponding equations for the variable $u$ read

$$
\hat{\tilde{u}}=\frac{\tilde{v}-\hat{v}}{\tilde{u}-\hat{u}}, \quad \overline{\tilde{u}}=\frac{\bar{v}-\tilde{v}}{\bar{u}-\tilde{u}}, \quad \overline{\hat{u}}=\frac{\bar{v}-\hat{v}}{\bar{u}-\hat{u}} .
$$

Successively, using equations (10), (12), we evaluate uniquely the updates

$$
(\hat{\tilde{u}}, \hat{\tilde{v}}), \quad(\overline{\tilde{u}}, \overline{\tilde{v}}), \quad(\overline{\hat{u}}, \overline{\hat{v}}),
$$

which are assigned to the vertices depicted with $\circ$. In a similar manner, from the updates (13) and the evolution of the lattice equations on the remaining three faces, we are free to obtain the values of the fields $(u, v, w)$ shifted in three directions, from three different ways. In principle, there is no particular reason for these three values to coincide, unless the P $\Delta \mathrm{E}$ 's (9) enjoy this special property. The confirmation of this test is crucial in that it verifies, at the first stage, the consistency of the embedding of the lattice BSQ into a multidimensional lattice. By simple and straightforward algebraic calculations, and eliminating all terms shifted in two directions in favour of terms 
shifted in one direction, one finds that

$$
\begin{aligned}
& \overline{\tilde{\tilde{u}}}=u+\frac{(\zeta-\sigma)(\tilde{u}-\bar{u})-(\zeta-\tau)(\hat{u}-\bar{u})}{(\hat{v}-\bar{v})(\tilde{u}-\bar{u})-(\tilde{v}-\bar{v})(\hat{u}-\bar{u})}, \\
& \overline{\hat{\tilde{v}}}=v+\frac{(\zeta-\sigma)(\tilde{u}-\bar{u}) \hat{u}-(\zeta-\tau)(\hat{u}-\bar{u}) \tilde{u}}{(\hat{v}-\bar{v})(\tilde{u}-\bar{u})-(\tilde{v}-\bar{v})(\hat{u}-\bar{u})} \\
& \overline{\hat{\tilde{w}}}=w+\frac{(\zeta-\sigma)(\tilde{v}-\bar{v})-(\zeta-\tau)(\hat{v}-\bar{v})}{(\hat{v}-\bar{v})(\tilde{u}-\bar{u})-(\tilde{v}-\bar{v})(\hat{u}-\bar{u})},
\end{aligned}
$$

independently of the way of calculating these values. This fact can be easily verified, since the fractional terms in equations (14), remain invariant under any permutation of the three lattice shifts, along with the corresponding lattice parameters.

Having established the three dimensional consistency of the lattice BSQ (using no more information than the equations themselves), it is now straightforward to derive the linear representation for the lattice BSQ system. This is accomplished by appointing $(\bar{u}, \bar{v})$ as projective variables, and identifying them with the ratio of homogeneous variables $\left(\psi_{0}, \psi_{1}, \psi_{2}\right)$, as follows

$$
\bar{u}=\frac{\psi_{1}}{\psi_{0}}, \quad \bar{v}=\frac{\psi_{2}}{\psi_{0}} .
$$

Substituting $(\bar{u}, \bar{v})$ given by equations (15), into the relevant equations (10), (12) and setting

$$
\tilde{\psi}_{0}=-\tilde{u} \psi_{0}+\psi_{1}, \quad \hat{\psi}_{0}=-\hat{u} \psi_{0}+\psi_{1}
$$

we obtain

$$
\begin{array}{ll}
\tilde{\psi}_{1}=-\tilde{v} \psi_{0}-\psi_{2}, & \tilde{\psi}_{2}=(\tau-\zeta+w \tilde{u}-u \tilde{v}) \psi_{0}-w \psi_{1}+u \psi_{2}, \\
\hat{\psi}_{1}=-\hat{v} \psi_{0}+\psi_{2}, & \hat{\psi}_{2}=(\sigma-\zeta+w \hat{u}-u \hat{v}) \psi_{0}-w \psi_{1}+u \psi_{2} .
\end{array}
$$

The linear system (16), (17) can be written in a matrix form as follows

$$
\tilde{\psi}=L \boldsymbol{\psi} \equiv\left(\begin{array}{ccc}
-\tilde{u} & 1 & 0 \\
-\tilde{v} & 0 & 1 \\
\triangleright & -w & u
\end{array}\right) \psi, \quad \hat{\psi}=M \psi \equiv\left(\begin{array}{ccc}
-\hat{u} & 1 & 0 \\
-\hat{v} & 0 & 1 \\
\triangleleft & -w & u
\end{array}\right) \psi
$$

where $\psi={ }^{t}\left(\psi_{0}, \psi_{1}, \psi_{2}\right)$, and the entries of the matrices $L$ and $M$ which are denoted by $\triangleright$ and $\triangleleft$, are uniquely determined by the relations

$$
\operatorname{det} L=\tau-\zeta, \quad \operatorname{det} M=\sigma-\zeta,
$$

respectively. It should be noted that the compatibility condition, $\hat{L} M=\tilde{M} L$, of the linear system (18) for every value of $\zeta$, holds on weaker equations than the lattice BSQ system (9). The validity of the previous compatibility condition on the solutions of the lattice BSQ, implies that the linear difference system (25), can be solved for every fixed value of $\zeta$. Thus, we may replace the column vector $\psi$, by a matrix $\Psi$ with values in $\operatorname{GL}(3, \mathbb{C})$, and which is formed by putting together three independent solutions of the system (18). 
4. The differential-difference compatible system. We now impose on $\Psi$, and subsequently on the fields, an additional dependence on the continuous lattice parameters $\tau, \sigma$. This will be done by imposing on the linear system (18), certain first order linear differential relations for $\Psi$, in such a way that the compatibility conditions

$$
\partial_{\tau} \tilde{\Psi}=\widetilde{\partial_{\tau} \Psi}, \quad \partial_{\sigma} \hat{\Psi}=\widehat{\partial_{\sigma} \Psi},
$$

are satisfied. Partial differentiation will be denoted by a comma, or by $\partial$, followed by the variable(s) with respect to which the differentiation has been performed. From the linear system (18), where now $\psi$ is replaced by the matrix $\Psi$, it follows that

$$
\operatorname{det} \Psi=(\tau-\zeta)^{n}(\sigma-\zeta)^{m}
$$

up to an insignificant constant which may be fixed to unity, without loss of generality. The previous observation and the matrix identity

$$
\operatorname{tr}\left(\Psi_{, \tau} \Psi^{-1}\right)=\frac{(\operatorname{det} \Psi)_{, \tau}}{\operatorname{det} \Psi}
$$

suggest that, a potential simple form of the first order linear differential equations for $\Psi$, is

$$
\Psi_{, \tau}=\frac{A}{\tau-\zeta} \Psi, \quad \Psi_{, \sigma}=\frac{B}{\sigma-\zeta} \Psi
$$

Here, $A$ and $B$ take values in $\operatorname{gl}(3, \mathbb{C})$, they do not depend on $\zeta$ and

$$
\operatorname{tr} A=n, \quad \operatorname{tr} B=m .
$$

The compatibility condition $\partial_{\tau} \tilde{\Psi}=\widetilde{\partial_{\tau} \Psi}$, for every value of $\zeta$, applied on the relevant equations (18), (23), leads to the system

$$
\begin{aligned}
G_{, \tau}+\Lambda+\Lambda A-\tilde{A} \Lambda & =0, \\
G A-\tilde{A} G & =0,
\end{aligned}
$$

where

$$
G=L-(\tau-\zeta) \Lambda
$$

and the matrix $\Lambda$ is given by

$$
\Lambda=\left(\begin{array}{lll}
0 & 0 & 0 \\
0 & 0 & 0 \\
1 & 0 & 0
\end{array}\right)
$$

After a lengthy elimination process applied to the system of equations (25), using the discrete operation, and taking also into account equation (9a) and the relevant trace relation from (24), one obtains the explicit form of the matrix $A$, and simultaneously the compatible differential-difference system. It turns out that the compatibility conditions (25) amount exactly to the equations obtained by identifying two different dyadic forms 
for the matrix $A$. Specifically, $A$ is given by

$$
A_{i j}=a_{i} a_{j}^{\prime}=c_{i} c_{j}^{\prime}
$$

where

$$
\begin{aligned}
& a_{i}=(1, \tilde{u}, \tilde{v}), \quad a_{i}^{\prime}=\left(n+\tilde{v} u_{, \tau}-\tilde{u} w_{, \tau}, w_{, \tau},-u_{, \tau}\right), \\
& c_{i}=\left(-u_{, \tau},-v_{, \tau}, n+\underline{w} u_{, \tau}-\underline{u} v_{, \tau}\right), \quad c_{i}^{\prime}=(\underline{w},-\underline{u}, 1),
\end{aligned}
$$

$i=0,1,2$, and $\dot{\sim}$ denotes backward shift in the lattice variable $n$. The identification of the two dyadic forms of $A$, leads to the following differential-difference equations $(\mathrm{D} \Delta \mathrm{E})$

$$
v_{, \tau}=\tilde{u} u_{, \tau}, \quad w_{, \tau}=\underline{u}_{, \tau}, \quad \tilde{v}+\underline{w}-\tilde{u} \underline{u}+\frac{n}{u_{, \tau}}=0 .
$$

The variables $v$ and $w$ can be easily eliminated from equations (30), leading to the following scalar $\mathrm{D} \Delta \mathrm{E}$ for the variable $u$

$$
\left(\frac{n}{u_{, \tau}}\right)_{, \tau}=(\tilde{u}-\underset{\sim}{u})_{\sim, \tau}^{u_{,}}+(\underset{\sim}{u}-\tilde{\tilde{u}}) \tilde{u}_{, \tau} .
$$

Since by construction the system is symmetric with respect to the lattice variables and the associated parameters, the replacement $(\tilde{*}, n, \tau)$ with $(\hat{*}, m, \sigma)$ in equations $(29)-(31)$, delivers the matrix $B$ and the associated $\mathrm{D} \Delta \mathrm{E}$ for the variables $(u, v, w)$. Explicitly, the matrix $B$ takes the dyadic expressions

$$
B=b_{i} b_{j}^{\prime}=d_{i} d_{j}^{\prime},
$$

where

$$
\begin{aligned}
& b_{i}=(1, \hat{u}, \hat{v}), \quad b_{i}^{\prime}=\left(m+\hat{v} u_{, \sigma}-\hat{u} w_{, \sigma}, w_{, \sigma},-u_{, \sigma}\right), \\
& d_{i}=\left(-u_{, \sigma},-v_{, \sigma}, m+w_{\wedge} u_{, \sigma}-u_{\lambda} v_{, \sigma}\right), \quad d_{i}^{\prime}=\left(w_{\wedge},-u_{1}, 1\right),
\end{aligned}
$$

$i=0,1,2$, and : denotes backward shift in the lattice variable $m$. The system of the compatible $\mathrm{D} \Delta \hat{\mathrm{Es}}$ is

$$
v_{, \sigma}=\hat{u} u_{, \sigma}, \quad w_{, \sigma}=u_{\wedge} u_{, \sigma}, \quad \hat{v}+\underset{\wedge}{w}-\hat{u} u_{\wedge}+\frac{m}{u_{, \sigma}}=0,
$$

which by eliminating the variables $v$ and $w$, leads to the following scalar $\mathrm{D} \Delta \mathrm{E}$ for $u$

$$
\left(\frac{m}{u_{, \sigma}}\right)_{, \sigma}=\left(\hat{u}-\underline{\lambda}_{\lambda}\right) u_{\lambda, \sigma}+(u-\hat{\hat{u}}) \hat{u}_{, \sigma} .
$$

The D $\Delta$ Es in equations (30) and (34), represent the hetero-Bäckund transformations between copies of the compatible set of PDEs, which we derive in the next section, for different values of the parameters $n$ and $m$. This issue, along with the derivation of auto-Bäcklund transformations of the continuous systems [9], will be the subject of a future publication. 
5. Compatible partial differential equations. We now reverse completely the role between the lattice variables $n, m$ and the lattice parameters $\tau, \sigma$, and we focus on the dependence of $\Psi$ and the fields $(u, v, w)$, on the continuous lattice parameters $\tau, \sigma \in \mathbb{C}$, only. In this way, fields which are assigned to different lattice vertices are interpreted as new dependent variables.

5.1. The generating PDE of the Boussinesq hierarchy. The compatibility condition $\Psi_{, \tau \sigma}=\Psi_{, \sigma \tau}$ on the linear system (23), for every value of $\zeta$, leads to the following system of matrix PDEs

$$
(\sigma-\tau) A_{, \sigma}+[A, B]=0, \quad(\sigma-\tau) B_{, \tau}+[A, B]=0 .
$$

Let us now choose the dyadic forms of $A$ and $B$, given by (29a) and (33a) respectively, and which for convenience we rewrite below, along with the relevant trace relations given by equations (24),

$$
\begin{array}{ll}
A_{i j}=a_{i} a_{j}^{\prime}, & a^{i} a_{i}^{\prime}=n, \\
B_{i j}=b_{i} b_{j}^{\prime}, & b^{i} b_{i}^{\prime}=m,
\end{array}
$$

$i, j=0,1,2$. Indices are raised and lowered using the Kronecker symbol $\delta_{i j}$, and summation over repeated upper and lower indices is understood. Inserting $A$ and $B$, given by (37) into equations (36), and after simple algebraic manipulations, taking into account the fact that $a_{0}=b_{0}=1$, we arrive at the following system of PDE's

$$
\begin{array}{ll}
(\tau-\sigma) a_{i, \sigma}-a^{j} b_{j}^{\prime}\left(a_{i}-b_{i}\right)=0, & (\tau-\sigma) a_{i, \sigma}^{\prime}+a^{j} b_{j}^{\prime} a_{i}^{\prime}-b^{j} a_{j}^{\prime} b_{i}^{\prime}=0, \\
(\tau-\sigma) b_{i, \tau}-b^{j} a_{j}^{\prime}\left(a_{i}-b_{i}\right)=0, & (\tau-\sigma) b_{i, \tau}^{\prime}+a^{j} b_{j}^{\prime} a_{i}^{\prime}-b^{j} a_{j}^{\prime} b_{i}^{\prime}=0,
\end{array}
$$

$i, j=0,1,2$. From the right set of the above of equations we find that $a_{i, \sigma}^{\prime}=b_{i, \tau}^{\prime}$. Using this fact, and the explicit form of $a_{i}^{\prime}$ and $b_{i}^{\prime}$, given by (29a) and (33a) respectively, we introduce the variables $\left(f_{0}, f_{1}, f_{2}\right)$ by the relations

$$
f_{1}=w, \quad f_{2}=-u, \quad f_{0, \tau}=n-a^{i} f_{i, \tau}, \quad f_{0, \sigma}=m-b^{i} f_{i, \sigma},
$$

$i=1,2$, and which satisfy

$$
a_{i}^{\prime}=f_{i, \tau}, \quad b_{i}^{\prime}=f_{i, \sigma},
$$

$i=0,1,2$. In terms of the new variables $f_{i}$, the system of equations (38) reads

$$
\begin{aligned}
(\tau-\sigma) a_{i, \sigma}-\left(m+\left(a^{j}-b^{j}\right) f_{j, \sigma}\right)\left(a_{i}-b_{i}\right) & =0, \\
(\tau-\sigma) b_{i, \tau}-\left(n-\left(a^{j}-b^{j}\right) f_{j, \tau}\right)\left(a_{i}-b_{i}\right) & =0, \\
(\tau-\sigma) f_{i, \tau \sigma}+m f_{i, \tau}-n f_{i, \sigma}+C_{i j}\left(a^{j}-b^{j}\right) & =0,
\end{aligned}
$$

where $i, j=1,2$. The components of the matrix $\left(C_{i j}\right)$, which is in general a rank two matrix, are defined as follows

$$
C_{i j}=f_{i, \tau} f_{j, \sigma}+f_{i, \sigma} f_{j, \tau}
$$

$i, j=1,2$. Solving the linear system (41c) for the differences $\left(a_{i}-b_{i}\right)$, and using the remaining field equations (41a), (41b), we obtain a fourth order coupled system of 
PDE's for the variables $\left(f_{1}, f_{2}\right)$, which is omitted because of its length. The latter system of PDEs are the Euler-Lagrange equations for the variational problem, associated with the Lagrangian density

$$
L=(\tau-\sigma) \frac{F E}{J^{2}}+m \frac{F}{J}+n \frac{E}{J} .
$$

Here, the scalar quantities $F, E$ and $J$ are given by

$$
F=\boldsymbol{f}_{, \tau \sigma} \times \boldsymbol{f}_{, \tau}, \quad E=\boldsymbol{f}_{, \tau \sigma} \times \boldsymbol{f}_{, \sigma}, \quad J=\boldsymbol{f}_{, \tau} \times \boldsymbol{f}_{, \sigma},
$$

where $\boldsymbol{f}$ is the vector with components $\left(f_{1}, f_{2}\right)$. In the following, we denote the parameter family of fourth order PDEs for the variables $\left(f_{1}, f_{2}\right)$, by $\boldsymbol{E}(L)=0$. We conclude this section by noting that the Lagrangian $L$ remains invariant, modulo $L$ and null Lagrangians, under the action of the projective group of linear fractional transformations on $\left(f_{1}, f_{2}\right)$, i.e.

$$
\left(f_{1}, f_{2}\right) \mapsto\left(\frac{\mathcal{A}_{11} f_{1}+\mathcal{A}_{12} f_{2}+\mathcal{A}_{13}}{\mathcal{A}_{31} f_{1}+\mathcal{A}_{32} f_{2}+\mathcal{A}_{33}}, \frac{\mathcal{A}_{21} f_{1}+\mathcal{A}_{22} f_{2}+\mathcal{A}_{23}}{\mathcal{A}_{31} f_{1}+\mathcal{A}_{32} f_{2}+\mathcal{A}_{33}}\right)
$$

where $\left(\mathcal{A}_{i j}\right) \in \operatorname{SL}(3, \mathbb{C})$, and under affine base transformations of the form

$$
(\tau, \sigma) \mapsto\left(\varepsilon_{1} \tau+\varepsilon_{2}, \varepsilon_{1} \sigma+\varepsilon_{2}\right), \quad \varepsilon_{1}, \varepsilon_{2} \in \mathbb{C}, \varepsilon_{1} \neq 0 .
$$

The symmetry transformations of $L$, given by (45) and (46), fall into the class of the socalled divergence symmetries of the associated variational problem. Every symmetry of this type is inherited as a Lie-point symmetry, by the corresponding Euler-Lagrange equations, see [10] for a detailed account on these topics.

5.2. The Boussinesq hierarchy. The coupled system of PDEs $\boldsymbol{E}(L)=0$ encodes the complete hierarchy of the Boussinesq equations. This can be illustrated by imposing on $\Psi$ and on the associated dependent variables, an additional dependence on an infinite set of indeterminates $x^{i}=\left(x^{1}, x^{2}, \ldots\right)$. Following the construction given in [11], [12], the dependence is given explicitly by certain (infinite) differential relations, in such a way that all these relations are mutually consistent to each other. To this end, we introduce the matrix $H$ with components

$$
H_{i j, \tau}=a_{i} f_{j, \tau}, \quad H_{i j, \sigma}=a_{i} f_{j, \sigma},
$$

$i, j=0,1,2$, and the matrix $Z$ by the relation

$$
Z=\left(\begin{array}{ccc}
0 & 1 & 0 \\
0 & 0 & 1 \\
-\zeta & 0 & 0
\end{array}\right)
$$

The existence of the matrix $H$ for the variables $(\tau, \sigma)$, may be easily verified by using the system of PDE's (41). In terms of the above matrices, the linear differential relations for $\Psi$ which can be simultaneously imposed on the linear system (23), are given by

$$
\begin{aligned}
& \partial_{1} \Psi=\left(Z+\left[\partial_{\zeta} Z, H\right]\right) \Psi, \\
& \partial_{2} \Psi=\left(Z^{2}+\left[\partial_{\zeta} Z^{2}, H\right]\right) \Psi,
\end{aligned}
$$




$$
\partial_{i+3} \Psi=-\left(\zeta \partial_{i}+\partial_{i} H\right) \Psi
$$

where $\partial_{i}=\partial_{x^{i}}$ and $i \in \mathbb{Z} \backslash 3 \mathbb{Z}$. Taking the compatibility condition $\partial_{1} \partial_{2} \Psi=\partial_{2} \partial_{1} \Psi$, on equations (49a) and (49b), we obtain a set of PDE's for the various entries of the matrix $H$. A straightforward differentiation and elimination process applied to the latter system, and identifying the main field variables $\left(f_{1}, f_{2}\right)$ using (47), we end up with the following set of PDE's

$$
\begin{aligned}
& \partial_{1} \partial_{2} f_{1}=2\left(\partial_{2}\right)^{2} f_{2}-3 \partial_{1}\left(\partial_{1} f_{2}\right)^{2}-\frac{1}{2}\left(\partial_{1}\right)^{2} \partial_{2} f_{2}+\frac{1}{2} \partial_{1} \partial_{2}\left(f_{2}^{2}\right)+\frac{1}{4}\left(\partial_{1}\right)^{4} f_{2}, \\
& \partial_{1} \partial_{1} f_{1}=\frac{1}{2} \partial_{1} \partial_{2} f_{2}+\frac{1}{2} \partial_{1}\left(\partial_{1} f_{2}\right)^{2}-\frac{1}{2}\left(\partial_{1}\right)^{3} f_{2} .
\end{aligned}
$$

Then, the final compatibility condition $\left(\partial_{1}\right)^{2} \partial_{2} f=\partial_{2}\left(\partial_{1}\right)^{2} f$ on the above system, leads to the Boussinesq equation

$$
3\left(\partial_{2}\right)^{2} \mathcal{Q}+\left(\partial_{1}\right)^{4} \mathcal{Q}+6\left(\partial_{1}\right)^{2}\left(\mathcal{Q}^{2}\right)=0
$$

where $\mathcal{Q}=-\partial_{1} f_{2}$. Similarly, higher members of the Boussinesq hierarchy can be derived from the system (49), in a systematic way.

5.3. The Ernst equations for an Einstein-Maxwell-Weyl field. Now we discuss various subsystems which are included in equations $\boldsymbol{E}(L)=0$. Such subsystems arise, by imposing additional algebraic or differential constraints on the space of the independent and the dependent variables and on the parameter space, in a consistent way with the equations $\boldsymbol{E}(L)=0$, or equivalently, with the auxiliary system (41).

Assuming first that the independent variables are real, one such consistent algebraic constraint is given by the following set of relations on the auxiliary variables,

$$
a_{1}=b_{1}+\frac{(\sigma-\tau) f_{1}^{\star}}{f_{2}+f_{2}^{\star}+f_{1} f_{1}^{\star}}, \quad a_{2}=b_{2}+\frac{\sigma-\tau}{f_{2}+f_{2}^{\star}+f_{1} f_{1}^{\star}},
$$

and on the parameters

$$
n+n^{\star}+1=0, \quad m+m^{\star}+1=0
$$

where $\star$ denotes complex conjugation. Imposing the constraints (52) and (53) on the auxiliary system (41), the latter reduces to a second order coupled system of PDEs for the main dependent variables $\left(f_{1}, f_{2}\right)$ solely, which is given by equations (41c). Relabeling the variables $\left(f_{1}, f_{2}\right)$ by $(\varphi, \mathcal{E})$, and using the language of forms, the latter set of PDEs can be written as

$$
\begin{aligned}
& \left(\mathcal{E}+\mathcal{E}^{\star}+\varphi \varphi^{\star}\right)(\mathrm{d}(\varrho * \mathrm{~d} \mathcal{E})-\mathrm{i} \boldsymbol{\beta} \wedge \mathrm{d} \mathcal{E})=2 \varrho\left(\mathrm{d} \mathcal{E}+\varphi^{\star} \mathrm{d} \varphi\right) \wedge * \mathrm{~d} \mathcal{E}, \\
& \left(\mathcal{E}+\mathcal{E}^{\star}+\varphi \varphi^{\star}\right)(\mathrm{d}(\varrho * \mathrm{~d} \varphi)-\mathrm{i} \boldsymbol{\beta} \wedge \mathrm{d} \varphi)=2 \varrho\left(\mathrm{d} \mathcal{E}+\varphi^{\star} \mathrm{d} \varphi\right) \wedge * \mathrm{~d} \varphi
\end{aligned}
$$

Here, * denotes the Hodge duality operator acting on the basis of one forms by

$$
* \mathrm{~d} \tau=\mathrm{d} \tau, \quad * \mathrm{~d} \sigma=-\mathrm{d} \sigma,
$$


while the function $\varrho$, and the one-form $\boldsymbol{\beta}$ are defined as

$$
\varrho=\frac{1}{2}(\sigma-\tau), \quad \boldsymbol{\beta}=v \mathrm{~d} \tau+\mu \mathrm{d} \sigma
$$

where $v, \mu \in \mathbb{R}$. The system of PDEs (54) forms the basis of the Einstein's field equations for plane symmetric spacetimes, in the presence of a source-free Maxwell field and a particular type Weyl neutrino field, and they are known in general relativity as the Ernst equations for an Einstein-Maxwell-Weyl field, [13]-[15].

6. Symmetry reduction to a second order system of ODEs. We are now interested in finding certain similarity solutions of equations $\boldsymbol{E}(L)=0$. This will be achieved by choosing a specific one-dimensional subgroup from the full group of Lie-point symmetries (45), (46). The requirement of invariance of the solutions under the onedimensional subgroup, leads to the corresponding similarity solutions.

Our motivation in finding such solutions is two-fold. First, one hopes to discover some explicit solutions of the fourth order coupled system $\boldsymbol{E}(L)=0$, which would provide physically significant solutions, in connection with the Ernst equations. In the present work however, we are interested mostly in writing down the system of the reduced ordinary differential equations (ODE), since the latter potentially define higher order analogous of the Painlevé ODE's.

To be more specific, we shall be interested in finding solutions of equations $\boldsymbol{E}(L)=$ 0 , which remain invariant along the orbits generated by the following vector field ${ }^{1}$

$$
X=\tau \partial_{\tau}+\sigma \partial_{\sigma}+2 \alpha_{1} f_{1} \partial_{f_{1}}+2 \alpha_{2} f_{2} \partial_{f_{2}}
$$

where $\alpha_{1}, \alpha_{2}$, arbitrary complex parameters. Requiring invariance, means that the functions $\left(f_{1}, f_{2}\right)$ should satisfy equations $\boldsymbol{E}(L)=0$, and also the compatible differential constraints

$$
\tau f_{i, \tau}+\sigma f_{i, \sigma}=2 \alpha_{i} f_{i}, \quad i=1,2,
$$

The general solution of the latter PDEs is

$$
f_{i}(\tau, \sigma)=F_{i}(t)(\tau \sigma)^{\alpha_{i}}, \quad t=\frac{\tau}{\sigma}, \quad i=1,2 . \quad \text { (no summation) }
$$

Inserting the main variables in the form given by equation (59), into equations $\boldsymbol{E}(L)=$ 0 , we obtain a fourth order system of ODEs for the functions $F_{i}$, which is omitted because of its length. However, an effective way in dealing with the latter system of ODEs, is to consider the auxiliary system (41). To this end, one needs first to find the vertical components of the vector field $X$, which are associated with the auxiliary variables $a_{i}, b_{i}$, and subsequently the consistent invariant form of the variables $a_{i}, b_{i}$. The corresponding symmetry generator $\mathcal{X}$ for the equations (41), is

$$
\mathcal{X}=X+\left(1-2 \alpha_{1}\right)\left(a_{1} \partial_{a_{1}}+b_{1} \partial_{b_{1}}\right)+\left(1-2 \alpha_{2}\right)\left(a_{2} \partial_{a_{2}}+b_{2} \partial_{b_{2}}\right)
$$

\footnotetext{
${ }^{1}$ The symmetry generator (57) generalizes the one that we used in [3], [16], to obtain a symmetry reduction of a scalar subsystem of equations $\boldsymbol{E}(L)=0$, to the full Painleve VI equation.
} 
which leads us to conclude that the invariant form of the auxiliary potentials is

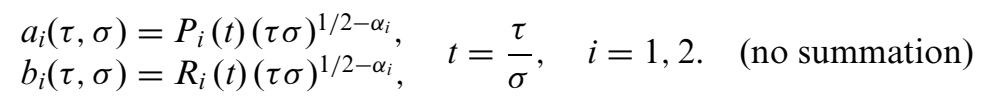

Substituting the dependent variables $a_{i}, b_{i}$ and $f_{i}$ given by (61) and (59), respectively, into equations (41), we obtain the following system of ODEs

$$
\begin{aligned}
& -(t-1)\left(t P_{i}^{\prime}-\left(\frac{1}{2}-\alpha_{i}\right) P_{i}\right)=\left(m-t^{1 / 2}\left(P^{j}-R^{j}\right)\left(t F_{j}^{\prime}-\alpha_{j} F_{j}\right)\right)\left(P_{i}-R_{i}\right), \\
& (t-1)\left(t R_{i}^{\prime}+\left(\frac{1}{2}-\alpha_{i}\right) R_{i}\right)=\left(n t-t^{1 / 2}\left(P^{j}-R^{j}\right)\left(t F_{j}^{\prime}+\alpha_{j} F_{j}\right)\right)\left(P_{i}-R_{i}\right), \\
& t^{2}(1-t) F_{i}^{\prime \prime}+t((n-1) t+m+1) F_{i}^{\prime}-\alpha_{i}\left(\left(n-\alpha_{i}\right) t-\left(m-\alpha_{i}\right)\right) F_{i} \\
& \quad=2 t^{1 / 2}\left(t^{2} F_{i}^{\prime} F_{j}^{\prime}-\alpha_{i} F_{i} \alpha_{j} F_{j}\right)\left(P^{j}-R^{j}\right),
\end{aligned}
$$

where prime denotes differentiation with respect to $t$. Employing a general quadratic ansatz on the first order derivatives of $F_{i}$ 's, we find that the system (62), admits two first integrals. Denoting the complex constants of integration by $s_{1}$ and $s_{2}$, these first integrals are given by

$$
\begin{aligned}
& \left(P_{1}-R_{1}\right)^{2}\left(t^{2} F_{1}^{\prime 2}-\alpha_{1}^{2} F_{1}^{2}\right)+\left(P_{1}-R_{1}\right)\left(P_{2}-R_{2}\right)\left(t^{2} F_{1}^{\prime} F_{2}^{\prime}-\alpha_{1} \alpha_{2} F_{1} F_{2}\right) \\
& \quad+t^{1 / 2}\left[\left(F_{1}^{\prime}+\frac{\alpha_{1}}{t} F_{1}\right)\left(\left(2 \alpha_{1}-1\right) P_{1}-m\left(P_{1}-R_{1}\right)\right)-t\left(F_{1}^{\prime}-\frac{\alpha_{1}}{t} F_{1}\right)\right. \\
& \left.\quad \times\left(\left(2 \alpha_{1}-1\right) R_{1}+n\left(P_{1}-R_{1}\right)\right)\right]+t\left(\alpha_{1} F_{1} F_{2}^{\prime}-\alpha_{2} F_{1}^{\prime} F_{2}\right) \\
& \quad \times\left[\left(P_{1} R_{2}-P_{2} R_{1}\right) \frac{\alpha_{1}+\alpha_{2}-1}{\alpha_{1}-\alpha_{2}}+\left(R_{1} R_{2}-P_{1} P_{2}\right)\right]=s_{1},
\end{aligned}
$$

together with the same relation, obtained from (63) by interchanging the subscripts $1 \leftrightarrow 2$. Through a straightforward elimination and differentiation process applied to the system (62), taking into account the first integrals and introducing new dependent variables $\left(\mathcal{G}_{1}, \mathcal{G}_{2}\right)$ by the substitutions,

$$
\mathcal{G}_{i}(t)=\frac{t}{\alpha_{i}} \frac{F_{i}^{\prime}(t)}{F_{i}(t)}, \quad i=1,2,
$$

we end up with a second order, coupled system of ODEs for the variables $\left(\mathcal{G}_{1}, \mathcal{G}_{2}\right)$, which involves six free parameters, namely $\left(n, m, \alpha_{1}, \alpha_{2}, s_{1}, s_{2}\right)$. In order to give an explicit form of the latter system of ODEs, we introduce the auxiliary quantities $\left(Q_{1}, Q_{2}\right)$ as follows

$$
\begin{aligned}
Q_{1}= & \frac{m+n t}{2 \alpha_{1}\left(\mathcal{G}_{1}-\mathcal{G}_{2}\right)}+\frac{(m-n t) \mathcal{G}_{2}}{2 \alpha_{1}\left(\mathcal{G}_{1}-\mathcal{G}_{2}\right)}+\frac{(t-1) \alpha_{2} \mathcal{G}_{1} \mathcal{G}_{2}\left(1-\mathcal{G}_{2}^{2}\right)}{2 \alpha_{1}\left(\mathcal{G}_{1}-\mathcal{G}_{2}\right)^{2}} \\
& -\frac{(t-1)\left(\alpha_{1}-\alpha_{2}\right)\left(\mathcal{G}_{2}^{2}-1\right)}{2 \alpha_{1}\left(\mathcal{G}_{1}-\mathcal{G}_{2}\right)^{2}}+\frac{(t-1) \mathcal{G}_{1}^{2}\left(\mathcal{G}_{2}^{2}-1\right)}{2\left(\mathcal{G}_{1}-\mathcal{G}_{2}\right)^{2}}+\frac{t(t-1)\left(\mathcal{G}_{2}^{2}-1\right) \mathcal{G}_{1}^{\prime}}{2 \alpha_{1}\left(\mathcal{G}_{1}-\mathcal{G}_{2}\right)^{2}} \\
& -\frac{t(t-1)\left(\mathcal{G}_{1} \mathcal{G}_{2}-1\right) \mathcal{G}_{2}^{\prime}}{2 \alpha_{1}\left(\mathcal{G}_{1}-\mathcal{G}_{2}\right)^{2}}
\end{aligned}
$$


and $Q_{2}$ is obtained from $Q_{1}$ by interchanging the subscripts $1 \leftrightarrow 2$. Then, the aforementioned system of ODEs is given by

$$
\begin{aligned}
& \frac{\alpha_{1} \alpha_{2}}{\alpha_{1}-\alpha_{2}}\left(\mathcal{G}_{1}-\mathcal{G}_{2}\right)\left(Q_{1} Q_{2}^{\prime}-Q_{1}^{\prime} Q_{2}\right)+\left((t-1) \mathcal{G}_{1}-(t+1)\right) Q_{1}^{\prime} \\
& -\frac{\alpha_{1}^{2}}{t}\left(\left(\mathcal{G}_{1}-1\right)^{2}-\frac{4 \mathcal{G}_{1}}{t-1}\right) Q_{1}^{2}-\frac{\alpha_{1} \alpha_{2}}{t}\left(\left(\mathcal{G}_{2}-1\right)^{2}-\frac{4 \mathcal{G}_{2}}{t-1}-\frac{\alpha_{1}\left(\mathcal{G}_{1}-\mathcal{G}_{2}\right)^{2}}{\alpha_{1}-\alpha_{2}}\right) Q_{1} Q_{2} \\
& -\frac{\alpha_{1}^{2}}{t}\left((t-1)\left(\mathcal{G}_{1}-1\right)^{2}-4 \mathcal{G}_{1}+\frac{t}{\alpha_{1}}\left(\mathcal{G}_{1}-1\right)+\frac{2 t}{\alpha_{1}} \frac{(n+m)}{(t-1)}\right) Q_{1}=s_{1}
\end{aligned}
$$

together with the same equation obtained from equation (66), by making the usual interchanges in the subscripts.

7. Perspectives. We finish our discussion by noting that, on the continuous level, the generating PDE of the Boussinesq hierarchy, namely the equations $\boldsymbol{E}(L)=0$, include the one of the $\mathrm{KdV}$ hierarchy. This is achieved by considering the case, where the dependent variables $\left(f_{1}, f_{2}\right)$ coincide, i.e. $f:=f_{1}=f_{2}$. The generating PDE of the $\mathrm{KdV}$ hierarchy was given in [3], and is the Euler-Lagrange equation for the variational problem associated with the Lagrangian

$$
\mathcal{L}=(\tau-\sigma) \frac{\left(f_{, \tau \sigma}\right)^{2}}{f_{, \tau} f_{, \sigma}}+\frac{1}{\tau-\sigma}\left(m^{2} \frac{f_{, \tau}}{f_{, \sigma}}+n^{2} \frac{f_{, \sigma}}{f_{, \tau}}\right)
$$

and which we denote by $\boldsymbol{E}(\mathcal{L})=0$. The Lagrangian $\mathcal{L}$ can be reduced from the Lagrangian $L$ given by equation (43), considering the aforementioned case, in a consistent way with the reduced equations. In [3], cf [16], it was shown that a specific symmetry reduction of the equations $\boldsymbol{E}(\mathcal{L})=0$ leads to invariant solutions, built from the full Painlevé VI equation. Thus, we expect that there exists an intimate connection of the six-parameter system of coupled ODEs, presented in Section 7, with the hierarchies of the Painlevé VI and the Garnier systems [17]. The latter issue along with the isomonodromy problem of the relevant ODEs, will be left to a future study.

The derivation of generating PDEs, which, in turn, provide a mechanism for obtaining new integrable systems, is a project of equal importance. To this end, the classification of scalar discrete integrable systems, obtained recently in [8], could serve as a starting point. However, some of the extra restrictions imposed on the classification scheme in [8], would exclude, in a strict sense, the discrete BSQ system (9). Thus, the classification of quadrilateral integrable discrete systems, in more than one dependent variable, seems to be a more complicated problem.

In the light of the intimate connection between the PDE's $\boldsymbol{E}(L)=0$ and $\boldsymbol{E}(\mathcal{L})=0$, and the Ernst equations, the former fourth order integrable systems acquire a certain physical importance. This stems from the fact that the solution space of the hyperbolic Ernst equations is embedded into the solution space of the fourth order systems. Consequently, it would be interesting to consider to what extent, solutions of the larger continuous systems and their discrete compatible systems, could provide new exact solutions of the Einstein's equations, for various physically significant problems. Such a problem is the head-on collision of plane fronted gravitational waves, coupled with electromagnetic and neutrino waves, see [18] and references therein. 
ACKNOWLEDGEMENTS. The work of A.T. was supported by the European postdoctoral fellowship Marie Curie, contract No HPMF-CT-2002-01639.

\section{REFERENCES} edition.

1. V. G. Kac, Infinite dimensional Lie algebras (Cambridge University Press, 1990), third

2. T. Miwa, M. Jimbo and E. Date, Solitons: Differential equations, symmetries and infinite dimensional algebras (Cambridge University Press, 2000).

3. F. Nijhoff, A. Hone and N. Joshi, On a Schwarzian PDE associated with the KdV, hierarchy Phys. Lett. A 267 (2000), 147-156.

4. F. W. Nijhoff, G. R. W. Quispel, J. Van der Linden and H. W. Capel, On some linear integral equations generating solutions of nonlinear partial differential equations, Physica 119A (1983), 101-142.

5. F. W. Nijhoff, V. G. Papageorgiou, H. W. Capel and G. R. W. Quispel, The lattice Gel'fand-Dikii hierarchy, Inverse Problems 8 (1992), 597-651.

6. A. J. Walker, Similarity reductions and integrable lattice equations, Ph.D. thesis (Leeds University, 2001).

7. F. W. Nijhoff and A. J. Walker, The discrete and continuous Painlevé VI hierarchy and the Garnier systems, Glasgow Math. J. 43A (2001), 109-123.

8. V. E. Adler, A. I. Bobenko and Yu. B. Suris, Classification of integrable equations on quad-graphs. The consistency approach, Commun. Math. Phys. 233 (2003), 513-543.

9. A. Tongas and F. Nijhoff, Generalized hyperbolic Ernst equations for an EinsteinMaxwell-Weyl field, J. Phys. A Math. Gen. 38 (2005), 895-906.

10. P. J. Olver, Equivalence, invariants, and symmetry (Cambridge University Press, 1995).

11. F. W. Nijhoff, Linear integral transformations and hierarchies of integrable nonlinear equations, Physica 31D (1988), 339-388.

12. F. W. Nijhoff, Integrable hierarchies, Lagrangian structures and non-commuting flows, in Topics in soliton theory and exactly solvable nonlinear equations (Oberwolfach, 1986), (World Sci. Publishing, Singapore, 1987), 150-181.

13. F. J. Ernst, New formulation of the axially symmetric gravitational field problem, Phys. Rev. 167 (1968), 1175-1178.

14. F. J. Ernst, New formulation of the axially symmetric gravitational field problem II, Phys. Rev. 168 (1968), 1415-1417.

15. G. A. Alekseev, Soliton configuration of interacting massless fields, Sov. Phys. Dokl. 28 (1983), 133-135.

16. A. Tongas, D. Tsoubelis and P. Xenitidis, A family of integrable nonlinear equations of hyperbolic type, J. Math. Phys. 42 (2001), 5762-5784.

17. R. Garnier, Sur des équations différentielles du troisième ordre dont l'intégrale générale est uniforme et sur une classe d'équations nouvelles d'ordre supérieur dont l'intégrale générale a ses points critiques fixes, Ann. Écol. Norm. Sup. 29 (1912), 1-126.

18. J. B. Griffiths, Colliding plane waves in general relativity (Oxford University Press, 1991). 
